\title{
Learning Materials Development of Parametric Curves and Surfaces for Modeling the Objects Using Maple on Differential Geometry Courses
}

\author{
Kusno*, Abduh Riski \\ Mathematics Department, Faculty of Mathematics and Sciences, University of Jember, Indonesia \\ ${ }^{*}$ Corresponding author. Email: kusno.fmipa@unej.ac.id
}

\begin{abstract}
Modeling industrial objects needs the formulas of curves and surfaces to construct a precise shape of real goods and simulate some process of form creations. For this reason, the study of the equations of curves and surfaces for objects modeling is essential for resulting in a required shape and feature of the goods. This study aims to enhance the instructional materials of differential geometry for fourth-semester college students. Learning materials help students to be able to design a real object using some parametric formulas of curves and surfaces with the software Maple. The method of research is as follows. (a) Instructional materials design for constructing objects; (b) Formulations and evaluations of graphs for goods modeling; (c) Modeling and simulating to realize these items. The research found some instructional materials and parametric formulas of curves and surfaces to equip students to design and evaluate real objects and home industry goods. The use of Maple can help them to present the graphs and the simulation process. The contributions of this study support the students to learn autonomously and creatively with their knowledge, technological skills, and experiences in implementing some differential geometry formulas (especially the curves and surfaces) for designing objects using the Maple tool.
\end{abstract}

Keywords: Development, Learning materials, Curves and surfaces, Modeling, Parametric formulas, Maple.

\section{INTRODUCTION}

Modeling industrial objects and some mechanical components can use curves and surfaces equations in the form of parametric representations [1,2]. Developing computer-aided design and manufacturing technologies needs the curves and surfaces formula to construct a precise shape of a real object and simulate some process of form creations. For example, using the curves can create many models of pipes [3-5], and the surfaces formulas can apply to design the shape of car bodies, electronic equipment, or cottage industry objects. On the other hand, we can implement the developable surfaces to draw surfaces of big objects constructed by plywood or plat-metal sheets, for example, aircraft, ship hulls industries, and trains [6-8]. For the reasons, the students learning formulation of curves and surfaces with software skills are vital to understanding the creating surfaces forms of the designed industrial objects [9-11]. It is to provide practical experience for the learners in modeling various industries' object shapes.
Object modeling is an activity to select, describe, and create a shape to meet a required function. This shape selection means the geometric modeling in the design process included graphics representations and analytic formulas of curves and surfaces. For this reason, it needs some equations of parametric curves and surfaces in the instructional materials of differential geometry that can use to create many different forms of surfaces of objects. The use of these formulas can produce an aesthetic shape of the designed goods. This paper discusses the development's learning materials for modeling the real goods and cottage industry objects using parametric representations supported with software-Maple.

This study aims to enhance the instructional materials of differential geometry for fourth-semester college students. Learning materials help students to be able to design a real object using some parametric formulas of curves and surfaces with the software Maple. This study contributes to students learning autonomously and creatively with their knowledge, technological skills, and 
experiences in implementing some differential geometry formulas (especially the curves and surfaces) for designing objects using the Maple tool.

\section{METHOD}

The method of research uses development research. This study deal with the understanding of learning, the design of learning contents, and the development of tools. The research results may find models, constructs, or instantiations that can significantly enhance the understanding of learning subjects matters $[12,13]$. In this case, we identify and explain the process of object design and development of teaching materials of the differential geometry courses using Maple [14,15]. The steps of research are as follows [16]. (a) Instructional materials design for constructing objects; (b) formulations and evaluations of graphs for goods modeling; (c) Modeling and simulating the industrial goods. The use of Maple is to support the students to obtain some design experiences during a simulation. Additionally, this software Maple may help the learners to immediate feedback to alter the model, re-test it quickly, and evaluate their modeling processes and results.

\section{RESULTS AND DISCUSSION}

\subsection{Instructional Materials Design for Constructing Objects}

We can present the parametric functions of curves and surfaces in $3 D$ respectively in form $\mathbf{C}(u): \mathscr{R}^{1} \rightarrow \mathscr{R}^{3}$ and $\mathrm{S}(\mathrm{u}, v): \mathscr{R}^{2} \rightarrow \mathscr{R}^{3}$ and the algebraic representation of these curves and surfaces are

$$
\begin{aligned}
& \mathbf{C}(u)=\langle x(u), y(u), z(u)\rangle=x(u) \mathbf{i}+y(u) \mathbf{j}+z(u) \mathbf{k} \\
& \mathbf{S}(u, v)=\langle x(u, v), y(u, v), z(u, v)\rangle= x(u, v) \mathbf{i}+ \\
& y(u, v) \mathbf{j}+z(u, v) \mathbf{k}
\end{aligned}
$$

with $x(u), y(u), z(u), x(u, v), y(u, v)$, and $z(u, v)$ the functions of real values. Developing instructional materials of the curves and the surfaces suitable with students' knowledge, learners skill in using Maple, and modeling objects form needs the investigation and the evaluation to the parametric formulas. Based on the instructional objectives of the differential geometry course, this research discusses to equip the students in designing the shapes of some industry objects. In this case, it evaluates some curves and surfaces formulas for constructing handicrafts and pipes shape.

\subsubsection{Some Parametric Presentations of Curves}

Some curves in the parametric form are presented as follows.
1. The formula of line segment $\overline{P Q}$ with the endpoints $P$ and $Q$ of the vectors position $\left.\mathbf{P}<x_{\mathrm{p}}, y_{\mathrm{p}}, z_{\mathrm{p}}\right\rangle$ and $\mathbf{Q}<x_{\mathrm{Q}}, y_{\mathrm{Q}}, z_{\mathrm{Q}}>$ is

$$
\mathbf{L}(u)=\left\langle(1-u) \cdot x_{\mathrm{p}}+u \cdot x_{\mathrm{Q}},(1-u) \cdot y_{\mathrm{p}}+u \cdot y_{\mathrm{Q}},\right.
$$

$$
(1-u) \cdot z_{\mathrm{p}}+u \cdot z_{\mathrm{Q}}>
$$

with $0 \leq u \leq 1$.

2. Consider two constant unity vectors perpendicular in space $\mathbf{u}_{1}=\left\langle a_{1}, a_{2}, a_{3}\right\rangle$ and $\mathbf{u}_{2}=\left\langle b_{1}, b_{2}, b_{3}\right\rangle$. It can define an ellipse laid in the plane $\left[\mathbf{u}_{1}, \mathbf{u}_{2}\right]$ of the center $\left\langle x_{\mathrm{C}}, y_{\mathrm{C}}\right.$, $z_{\mathrm{C}}>$ in the expression

$$
\mathbf{E}(u)=\left\langle x_{\mathrm{C}}, y_{\mathrm{C}}, z_{\mathrm{C}}\right\rangle+\left[p \cdot \cos \varphi \mathbf{u}_{1}+q \cdot \sin \varphi \mathbf{u}_{2}\right]
$$

with $p$ and $q$ positive real values, $\varphi=2 \pi u$ and $0 \leq u \leq 1$. If the curves are a circle, a limacon, and a rose, then it can define the parametric formulas respectively [3]

$$
\begin{aligned}
& \mathbf{C}_{1}(u)=r_{1}(u) \cdot\left[\cos \varphi \mathbf{u}_{1}+\sin \varphi \mathbf{u}_{2}\right] \\
& \mathbf{C}_{2}(u)=r_{2}(u) \cdot\left[\cos \varphi \mathbf{u}_{1}+\sin \varphi \mathbf{u}_{2}\right] \\
& \mathbf{C}_{3}(u)=r_{3}(u) \cdot\left[\cos \varphi \mathbf{u}_{1}+\sin \varphi \mathbf{u}_{2}\right]
\end{aligned}
$$

with the real functions $r_{1}(u)=p ; r_{2}(u)=p \pm q \cdot \sin \varphi$ or $r_{2}(u)=p \pm q \cdot \cos \varphi ; r_{3}(u)=p \pm q \cdot \sin (n . \varphi)$ or $r_{3}(u)=p \pm$ $q . \cos (n . \varphi)$. In this case, the values $p$ and $q$ are positive real constants, $\varphi=2 \pi . u$ and $0 \leq u \leq 1$, with the number of rose leafs $n$.

3. The heliks with the center $\left(x_{1}, y_{1}, z_{1}\right)$ is

$$
\mathbf{H}_{\mathrm{L}}(u)=\left\langle x_{1}+p \cdot \cos u, y_{1}+q \cdot \sin u, z_{1}+u\right\rangle .
$$

4. Let a regular curve $\mathbf{C}(u)$ of Equation (1a) differentiated twice in interval $0 \leq u \leq 1$. In the natural parameter $s$, the tangent unit vector $\mathbf{t}$, the normal unit vector $\mathbf{n}$, and the unit binormal vector $\mathbf{b}$ of the curve $\mathbf{C}(u)$ are orthogonal to each other in the form [5]

$$
\begin{aligned}
& \mathbf{t}=\frac{d \mathbf{C}(u)}{d s}=\dot{\mathbf{C}}=\frac{\mathbf{C}^{\prime}}{\left|\mathbf{C}^{\prime}\right|} \\
& \mathbf{n}=-\frac{\mathbf{k}}{|\mathbf{k}|} \text { and } \mathbf{k}=\frac{\mathbf{d t}}{\mathbf{d s}}=\dot{\mathbf{t}} \\
& \mathbf{b}=\mathbf{t} \wedge \mathbf{n} .
\end{aligned}
$$

\subsubsection{Some Parametric Presentations of Surfaces}

Some surfaces in the parametric form are presented as follows.

1. The parametric formula of sphere or ellipsoid of cente $\left\langle x_{\mathrm{C}}, y_{\mathrm{C}}, z_{\mathrm{C}}\right\rangle$ is successively

$$
\begin{array}{r}
\mathbf{S}_{p}(u, v)=\left\langle r \cdot \sin u \cos v+x_{\mathrm{C}}, r \cdot \sin u \cdot \sin v+y_{\mathrm{C}},\right. \\
\left.r \cdot \cos u+z_{\mathrm{C}}\right\rangle
\end{array}
$$

$$
\mathbf{E}(u, v)=\left\langle r_{1} \cdot \sin u \cos v+x_{\mathrm{C}}, r_{2} \cdot \sin u \cdot \sin v+y_{\mathrm{C}},\right.
$$$$
r_{3} \cdot \cos u+z_{\mathrm{C}}>
$$

(7b)

with $r, r_{1}, r_{2}$, and $r_{3}$ positive real constants.

2 . The cylinder of radius $r$ with the axe $\left(x_{\mathrm{C}}, y_{\mathrm{C}}, z_{\mathrm{C}}\right)$ is 
(8)

$$
\mathbf{C}_{\mathrm{r}}(u, v)=\left\langle r \cdot \cos u+x_{\mathrm{C}} \cdot v, r \cdot \sin u+y_{\mathrm{C}} \cdot v, z_{\mathrm{C}} \cdot v\right\rangle
$$

with $r$ positive real.

3. The hyperboloid of center $\left\langle x_{\mathrm{C}}, y_{\mathrm{C}}, z_{\mathrm{C}}\right\rangle$ is

$$
\begin{array}{r}
\mathbf{H}(u, v)=\left\langle(\cos u-v \cdot \sin u)+x_{\mathrm{C}},\right. \\
(\sin u+v \cdot \cos u)+y_{\mathrm{C}}, v+z_{\mathrm{C}}>.
\end{array}
$$

4. The paraboloid of radius $r$ and center $\left\langle x_{\mathrm{C}}, y_{\mathrm{C}}, z_{\mathrm{C}}\right\rangle$ is

$\mathbf{P}(u, v)=<r \cdot v \cdot \cos u+x_{\mathrm{C}}, r \cdot v \cdot \sin u+y_{\mathrm{C}}$,

$$
r_{1} \cdot v^{2}+z_{\mathrm{C}}>\text {. }
$$

with $r, r_{l}$ positive real constants.

5. The cone of radius $r$ and center $\left\langle x_{\mathrm{C}}, y_{\mathrm{C}}, z_{\mathrm{C}}\right\rangle$ is

$\mathbf{K}(u, v)=\left\langle r \cdot v \cdot \cos u+x_{\mathrm{C}}, r \cdot v \cdot \sin u+y_{\mathrm{C}}\right.$

$$
r_{1} \cdot v+z_{\mathrm{C}}>\text {. }
$$

with $r, r_{l}$ positive real constants.

6 . The circle-plane of radius $r$, height $z_{1}$, and center $\left\langle x_{\mathrm{C}}, y_{\mathrm{C},}, z_{\mathrm{C}}\right\rangle$ is

$$
\mathbf{C}_{\mathrm{L}}(u, v)=\left\langle r . u \cdot \cos v+x_{\mathrm{C}}, r . u \cdot \sin v+y_{\mathrm{C}}, z_{\mathrm{C}}\right\rangle .
$$

7. The pseudo-sphere of radius $r$ is

$\mathbf{P}_{\mathrm{B}}(u, v)=\left\langle r / v \cdot \cos u, r / v \cdot \sin u, r_{1} . v\right\rangle$. with $r, r_{1}$ positive real constants.

\subsection{Formulations and Evaluations of Graphs for Objects Modeling}

Helping students plot the graphs and implement Equation 1 up to Equation 15 to design real objects is necessary for the learners to evaluate these curves and surfaces formula using software-Maple $[11,17,18]$. Then, they try to create more complex graphs by connecting some curves and surfaces for modeling the object shapes. This section will study Equation 1-15 deals with the presentation aspects of the curves and surfaces, and the formulation method with Maple.

Line segment formula of Equation 2 can directly plot the line segment $\overline{P Q}$ from the vector positions $\mathbf{P}<x_{\mathrm{p}}, y_{\mathrm{p}}$, $z_{\mathrm{p}}>$ to the vector position $\mathbf{Q}<x_{\mathrm{Q}}, y_{\mathrm{Q}}, z_{\mathrm{Q}}>$ without tangent line calculation and determination of paramater value $u$. For example, when the vector position of the endpoint points $P$ and $Q$ are respectively $\mathbf{P}<5,2,1>$ and $\mathbf{Q}<0,8,6>$, it finds Figure 1a. Then, using Formula 3 and 4 can define an ellips, a circle, a limacon, and a rose that are laid in the plane of unity vectors $\mathbf{u}_{1}=\left\langle a_{1}, a_{2}, a_{3}\right\rangle$ and $\mathbf{u}_{2}$ $=\left\langle b_{1}, b_{2}, b_{3}\right\rangle$. The plotted shapes, generally, are symmetric and closed curves. These curves type usually use to construct the shapes of flower vases' rack, plane decorations, and model various forms of pipes crosssection. If we pose $\mathbf{u}_{1},=\langle\langle 3 / 5,4 / 5,0\rangle\rangle, \mathbf{u}_{2}=\langle 0,0,1\rangle$ and the Equation (3) is in the form $\mathbf{E}(u)=\langle 2,2,2\rangle+[5$. $\left.\cos \varphi \mathbf{u}_{1}+8 . \sin \varphi \mathbf{u}_{2}\right]$, then it gets Figure 1b. When Equations $4 \mathrm{a}, 4 \mathrm{~b}$, and $4 \mathrm{c}$ are $\mathbf{C}_{1}(u)=6 \cdot\left[\cos \varphi \mathbf{u}_{1}+\sin \varphi\right.$ $\left.\mathbf{u}_{2}\right], \mathbf{C}_{2}(u)=(2+4 \cdot \cos \varphi) \cdot\left[\cos \varphi \mathbf{u}_{1}+\sin \varphi \mathbf{u}_{2}\right]$, and $\mathbf{C}_{3}(u)$ $=(3+\cos (3 . \varphi)) \cdot\left[\cos \varphi \mathbf{u}_{1}+\sin \varphi \mathbf{u}_{2}\right]$, it can obtain Figure $1 \mathrm{c}, 1 \mathrm{~d}$, and 1e, respectively. In case the helix formulation of Equation 5 in the form $\mathrm{H}_{\mathrm{L}}(u)=<2+4 \cdot \cos u, 1+6 \cdot \sin$ $u, 3+u>$ with $0 \leq u \leq 4 \pi$, it will give Figure $1 \mathrm{f}$. On the other hand, consider a curve $\mathbf{C}(u)=<17 u^{3}-30 u^{2}+6 u$ $12,-39 u^{3}+51 u^{2}-36 u+10,203 u^{3}+393 u^{2}-195 u+15>$ and the parameter $u$ in interval $0 \leq u \leq 1$. We can draw the curve $\mathbf{C}(u)$ in the form of Figure $1 \mathrm{~g}$ and calculate the tangent vectors $\mathbf{t}$ of the curve $\mathbf{C}(u)$ at $u=0$ and $u=1$ as shown in Figure 1h. These defined parametric curves in Figure 1a-1h are presented using Maple with the syntaxes as shown in Table 1 .

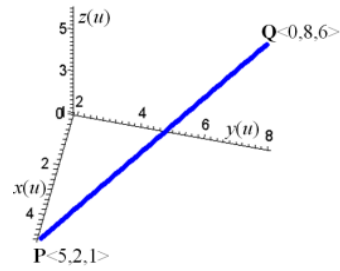

(a)

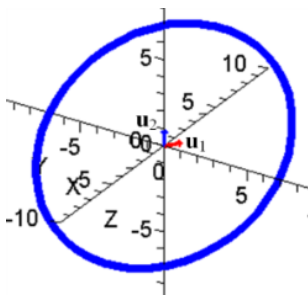

(c)

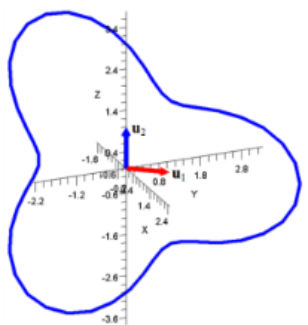

(e)

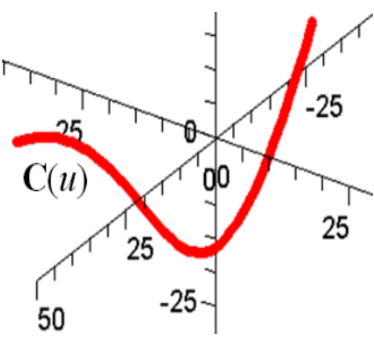

$(\mathrm{g})$

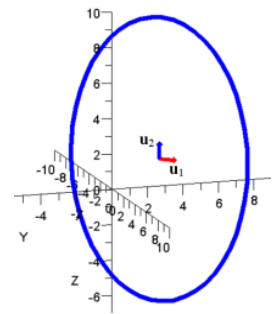

(b)

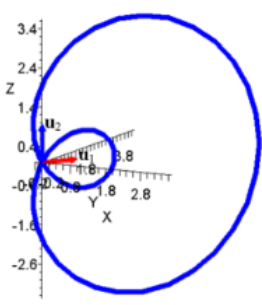

(d)

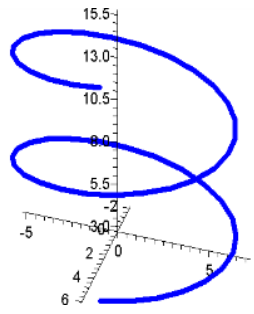

(f)

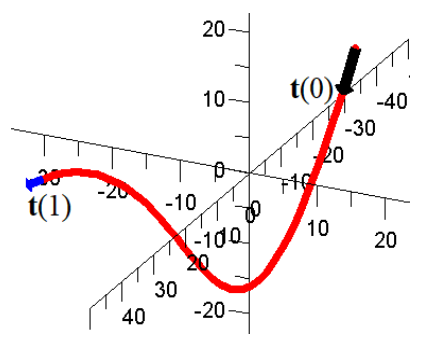

(h)
Figure 1 Some parametric curves. 
Table 1. Plotting the parametric curves using Maple

\begin{tabular}{|c|c|c|}
\hline No. & Equations Representation of Figures 1a-1h Using Maple & Explanations \\
\hline 1. & $\begin{array}{l}>\text { with(plots): } \\
>\text { spacecurve }\left(\left[(1-u)^{*} 5+u * 0,(1-u)^{*} 2+8 * u, 1-u+6 * u\right], u=0 . .1, \text { thickness }=3 \text {, }\right. \\
\quad \text { color = blue })\end{array}$ & Figure 1a \\
\hline 2. & 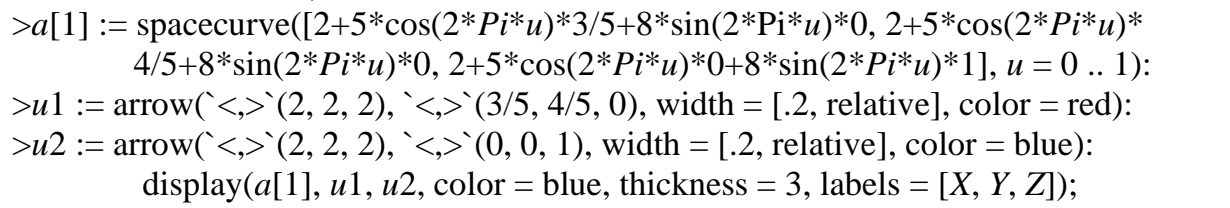 & Figure $1 b$ \\
\hline 3. & $\begin{aligned}>a[2]:= & \operatorname{spacecurve}\left(\left[7 *\left(\cos \left(2 * P i^{*} u\right) * 3 / 5+\sin \left(2 * P i^{*} u\right) * 0\right), 7 *\left(\cos \left(2 * P i^{*} u\right)^{*} 4 / 5+\right.\right.\right. \\
& \left.\left.\left.\sin \left(2 * \mathrm{Pi}^{*} \mathrm{u}\right) * 0\right), 7 *\left(\cos \left(2 * \mathrm{Pi}^{*} \mathrm{u}\right) * 0+\sin \left(2 * \mathrm{Pi}^{*} \mathrm{u}\right) * 1\right)\right], \mathrm{u}=0 \ldots 1\right): \\
>a[3]:= & \operatorname{spacecurve}\left(\left[\left(2+4 * \cos \left(2 * P i^{*} u\right)\right)^{*}\left(\cos \left(2 * P i^{*} u\right)^{*} 3 / 5+\sin \left(2 * P i^{*} u\right) * 0\right),(2+\right.\right.\end{aligned}$ & Figure 1c \\
\hline 4. & $\begin{array}{l}\left.4^{*} \cos \left(2 * P i^{*} u\right)\right)^{*}\left(\cos \left(2 * P i^{*} u\right)^{*} 4 / 5+\sin \left(2 * P i^{*} \mathrm{u}\right) * 0\right),\left(2+4 * \cos \left(2 * P i^{*} u\right)\right)^{*} \\
\left.\left.\left(\cos \left(2 * P i^{*} u\right)^{*} 0+\sin \left(2 * P i^{*} u\right)^{*} 1\right)\right], u=0 . .1\right)\end{array}$ & Figure 1d \\
\hline 5. & $\begin{aligned}>a[4]:= & \operatorname{spacecurve}\left(\left[\left(3+\cos \left((3 * 2) * P i^{*} u\right)\right)^{*}\left(\cos \left(2^{*} P i^{*} u\right)^{*} 3 / 5+\sin \left(2 * P i^{*} u\right)^{*} 0\right)\right.\right. \\
& \left(3+\cos \left(\left(3^{*} 2\right)^{*} P i^{*} u\right)\right)^{*}\left(\cos \left(2^{*} P i^{*} u\right)^{*} 4 / 5+\sin \left(2^{*} P i^{*} u\right)^{*} 0\right),\left(3+\cos \left(\left(3^{*} 2\right)^{*}\right.\right. \\
& \left.\left.\left.\left.P i^{*} u\right)\right)^{*}\left(\cos \left(2 * P i^{*} \mathrm{u}\right) * 0+\sin \left(2^{*} P i^{*} u\right)^{*} 1\right)\right], u=0 \ldots 1\right)\end{aligned}$ & Figure 1e \\
\hline 6. & $>$ spacecurve $([2+4 * \cos (u), 1+6 * \sin (u), 3+u], \mathrm{u}=0 . .4 * P i$, thickness $=3$, color $=$ blue $)$ & Figure $1 \mathrm{f}$ \\
\hline 7. & $\begin{array}{l}\text { >spacecurve }\left(\left[\left(17^{*} u^{*} u\right)^{*} u-30^{*} u^{*} u+60^{*} u-12,-\left(39^{*} u^{*} u\right)^{*} u+51^{*} u^{*} u-36^{*} u+10,\right.\right. \\
\left.\quad-\left(203 * u^{*} u\right)^{*} u+393^{*} u^{*} u-195^{*} u+15\right], u=0 \ldots 1, \text { thickness }=3 \text {, color }=\text { red, } \\
\quad \text { view }=[-50 \ldots 50,-50 \ldots 50,-50 \ldots 50])\end{array}$ & Figure $1 \mathrm{~g}$ \\
\hline 8. & 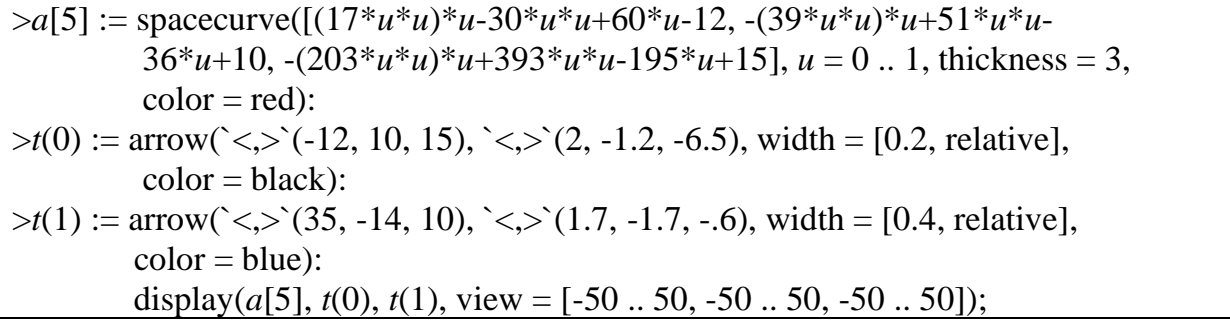 & Figure $1 \mathrm{~h}$ \\
\hline
\end{tabular}

From Equation 7 to the Equation 13, it can demonstrate the parametric surfaces in this ways. The sphere and ellipsoid in Equation 7 of center $\langle 3,2,4\rangle$ with the radius $r=6, r_{1}=3, r_{2}=5, r_{3}=8$, and $0 \leq u \leq 1.8 \pi$, and $0 \leq v \leq \pi$ produce the shapes in Figure $2 \mathrm{a}$ and $2 \mathrm{~b}$. Then, the cylinder of Equation 8 with the radius $r=5$, the axe $(0.5,2,2), 0 \leq u \leq 2 \pi$, and $0 \leq v \leq 12$ presents Figure 2c. Formulation of hyperboloid, paraboloid, cone, and circle-plane of Equation 9, 10, 11, and 12 in the forms $\mathbf{H}(u, v)=<(2 \cdot \cos \mathrm{u}-2 \cdot v \cdot \sin \mathrm{u})+2,(2 \cdot \sin u+2 . v \cdot \cos u)$ $+2,3 . v+3>$ with $0 \leq u \leq 2 \pi$ and $-3 \leq v \leq 3, \mathbf{P}(u, v)=<$ 3. v. $\cos u+1,3 \cdot v \cdot \sin u+2,2 \cdot v^{2}+3>$ with $0 \leq u \leq 2 \pi$ and $-3 \leq v \leq 3, \mathbf{K}(u, v)=<5$. v. $\cos u+2,5$. v. $\sin u+3,7 \cdot v+$ $1>$ with $0 \leq u \leq 2 \pi$ and $0 \leq v \leq 5$, and $\mathbf{C}_{\mathbf{L}}(u, v)=<6$. v.cos $u+1,6 . v \cdot \sin u+3,4>$ with $0 \leq u \leq 2 \pi$, and $0 \leq v \leq 1$ give the surfaces presented in Figure $2 \mathrm{~d}, 2 \mathrm{e}, 2 \mathrm{f}$, and $2 \mathrm{~g}$, successively. Finally, the pseudo-sphere of formula $\mathbf{P}_{\mathbf{B}}(u, v)=\langle 12 / v \cdot \cos u, 12 / v \cdot \sin u, 5 . v>$ with $-\pi \leq \mathrm{u} \leq \pi$ and $-1 \leq \mathrm{v} \leq-5$ presents the Figure $2 \mathrm{~h}$.

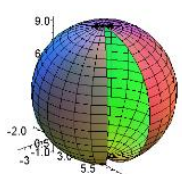

(a)

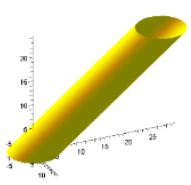

(c)

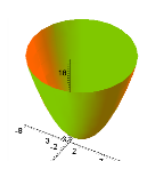

(e)

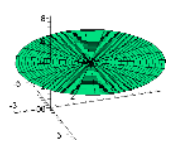

(g)

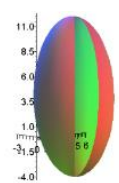

(b)

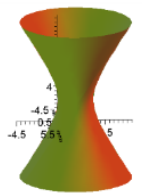

(d)

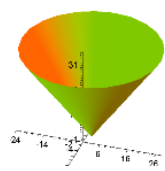

(f)

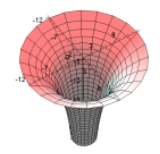

(h)
Figure 2 Some parametric surfaces. 
Table 2. Plotting the parametric surfaces using Maple

\begin{tabular}{|c|c|c|}
\hline No. & Equations Representation of Figures 2a-2h Using Maple & Explanations \\
\hline 1. & $\begin{array}{l}>\text { with(plots): } \\
>\operatorname{plot} 3 \mathrm{~d}\left(\left[5^{*} \sin (v) * \cos (u)+3,5^{*} \sin (v)^{*} \sin (u)+2,5^{*} \cos (v)+4\right], u=0 . .1 .8^{*} P i, v=0 . . P i\right):\end{array}$ & Figure $2 \mathrm{a}$ \\
\hline 2. & $>\operatorname{plot} 3 \mathrm{~d}\left(\left[3^{*} \sin (v)^{*} \cos (u)+3,5^{*} \sin (v)^{*} \sin (u)+2,8^{*} \cos (v)+4\right], u=0 . .1 .8^{*} P i, v=0 . . P i\right):$ & Figure $2 b$ \\
\hline 3. & $>\operatorname{plot} 3 \mathrm{~d}\left(\left[5^{*} \cos (u)+0.5^{*} v, 5^{*} \sin (u)+2 * v, 2 * v\right], u=0 . .2 * P i, v=0 . .12\right)$ & Figure $2 \mathrm{c}$ \\
\hline 4. & $\begin{array}{l}>\operatorname{plot} 3 \mathrm{~d}\left(\left[2 * \cos (u)-2 * v^{*} \sin (u)+2,2 * \sin (u)+2 * v^{*} \cos (u)+2,3 * v+3\right], u=0 \ldots 2 * P i\right. \\
\quad v=-3 . .3)\end{array}$ & Figure $2 d$ \\
\hline 5. & $>\operatorname{plot} 3 \mathrm{~d}\left(\left[3 * v^{*} \cos (u)+1,3 * v^{*} \sin (u)+2,2 * v^{*} v+3\right], u=0 . .2 * P i, v=-3 . .3\right):$ & Figure $2 \mathrm{e}$ \\
\hline 6. & $>\operatorname{plot} 3 \mathrm{~d}\left(\left[5^{*} v^{*} \cos (u)+2,5^{*} v^{*} \sin (u)+3,7 * v+1\right], u=0 \ldots 2 * P i, v=0 \ldots 5\right)$ & Figure $2 f$ \\
\hline 7. & $>\operatorname{plot} 3 \mathrm{~d}\left(\left[6^{*} v^{*} \cos (u)+1,6^{*} v^{*} \sin (u)+3,4\right], u=0 . .2 * P i, v=0 . .1\right)$ & Figure $2 \mathrm{~g}$ \\
\hline 8. & $>\operatorname{plot} 3 \mathrm{~d}\left(\left[(12 / v) * \cos (u),(12 / v)^{*} \sin (u), 5^{*} v\right], u=-P i \ldots P i, v=-1 \ldots-5\right)$ & Figure $2 \mathrm{~h}$ \\
\hline
\end{tabular}

Consider two parametric curves of Equation 1a in the forms $\mathbf{C}_{1}(u)$ and $\mathbf{C}_{2}(u)$ with $0 \leq u \leq 1$. The connection of both curves are respectively a parametric continuity order 0 and 1, i.e., $C^{0}$ and $C^{1}$, if they meet $\mathbf{C}_{1}(1)=\mathbf{C}_{2}(0)=\mathbf{C}_{P}$ and $\mathbf{C}_{1}^{\prime}(1)=\mathbf{C}_{2}^{\prime}(0)$. If the condition of tangent vectors $\mathbf{C}_{1}^{\prime}(1)$ and $\mathbf{C}_{2}^{\prime}(0)$ are collinear, it fulfills the geometric continuity. In case the surfaces $\mathbf{S}_{1}(u, v)$ and $\mathbf{S}_{2}(u, v)$ with $0 \leq u, v \leq 1$, they have to fulfill $\mathbf{S}_{1}(1, v)=\mathbf{S}_{2}(0, v)=\boldsymbol{\rho}(v)$ and $\mathbf{S}_{1}^{u}(1, v)=\mathbf{S}_{2}^{u}(0, v)$ along the common curve $\boldsymbol{\rho}(v)$.

Connection of these parametric curves and surfaces can use to model the shape of an object. So, it needs the calculation of common boundary point $\mathbf{C}_{P}$ and the computation of collinear tangent vectors $\mathbf{C}_{1}^{\prime}(1)=\mathbf{C}_{2}^{\prime}(0)$ of the curves joints. On the other sides, we have to evaluate the common boundary curve $\boldsymbol{\rho}(v)$ and the derivation in direction $u$ of the surfaces $\mathbf{S}_{1}^{u}(1, v)=\mathbf{S}_{2}^{u}(0, v)$ or the unique tangent plane along the common curve $\boldsymbol{\rho}(v)$ of the surfaces joints. For examples, let $\mathbf{C}_{1}(u)=\left\langle 2 u^{2}, 2 u-1\right.$, $3 u-2\rangle, \mathbf{C}_{2}(u)=\left\langle 2 u^{2}+4 u+2,2 u^{3}+2 u+1,3 u+1>\right.$ and $0 \leq u \leq$ 1. We can calculate the common boundary point $\mathbf{C}_{1}(1)$ $=\mathbf{C}_{2}(0)=\langle 2,1,1\rangle$, and the common tangent vector $\mathbf{C}_{1}^{\prime}(1)$ $=\mathbf{C}_{2}^{\prime}(0)=\langle 4,2,3\rangle$ as shown in Figure $3 \mathrm{a}$ and $3 \mathrm{~b}$.

When the surfaces $\mathbf{S}_{1}(u, v)$ and $\mathbf{S}_{2}(u, v)$ are $\mathbf{S}_{1}(u, v)=$ $<\left(2 u^{2}+2\right) \cdot \cos (2 \pi v),\left(2 u^{2}+2\right) \cdot \sin (2 \pi v), 4 u+1>$ and $\mathbf{S}_{2}(u, v)=$ $<\left(2 u^{2}+4 u+4\right) \cdot \cos (2 \pi v), \quad\left(2 u^{2}+4 u+4\right) \cdot \sin (2 \pi v)$, $2 u^{3}+u^{2}+4 u+5>$ with $0 \leq u, v \leq 1$, we find $\mathbf{S}_{1}(1, v)=\mathbf{S}_{2}(0, v)$ $=\boldsymbol{\rho}(v)=\langle 4 \cdot \cos (2 \pi v), 4 \cdot \sin (2 \pi v), 5\rangle$ as shown in Figure $3 c$. The derivation in direction $u$ of both surafces along the common boundary curve $\boldsymbol{\rho}(v)$ is $\mathbf{S}_{1}^{u}(1, v)=\mathbf{S}_{2}^{u}(0, v)=$ $<4 \cdot \cos (2 \pi v), 4 \cdot \sin (2 \pi v), 4\rangle$. The connection of these surfaces is presented in Figure $3 \mathrm{~d}$.

The parametric curves and surfaces formulas of Equation 2-12 are demonstrated in Table 1 and Table 2 using Maple. These formulas may give some advantages to model various forms of home industry objects. Then, joining the curves and surfaces may also are used to construct the object components and the required goods.
Based on these studies, we discuss, in the next section, the construction of the cottage industry and pipes.

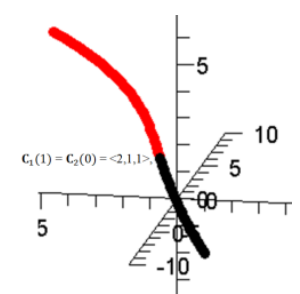

(a)

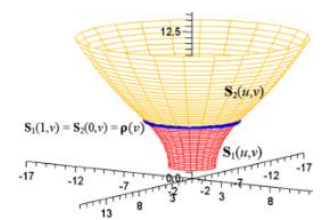

(c)

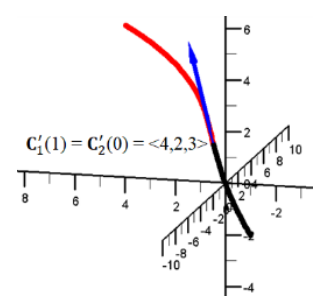

(b)

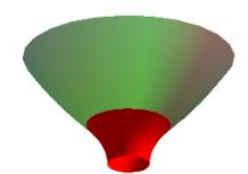

(d)
Figure 3 Connection of parametric curves and surfaces.

\subsection{Modeling and Simulating Industrial Objects}

\subsubsection{Design of Home Industrial Objects}

We show the use of these curves for creating iron trellis and frames, for example, to construct the flower vases' rack. Making the designed rack be symmetric and balanced form, it can reference from a cube or rectangular parallelepiped shapes $A B C D-E F G H$ as follows. From Figure 4a, we determine the line segments $K A=D K=K L$, $P B=P C=P O$, and $L M=M N=N O$. In the plane $K L O P$, it alternately draws the semicircles with diameter $L M, M N$, and $N O$. In-plane $A E H D$ and $B F G C$, it can plot the semicircles of radius $K A$ and $P B$ with the center $K$ and $P$, respectively, as presented in Figure $4 \mathrm{~b}$. Then, it can pose some circles with centers at the points $L, M, N, O$, and the summit points of the semicircles to provide the places of flower vases. The design result may introduce in Figure $4 \mathrm{c}, 4 \mathrm{~d}$ 


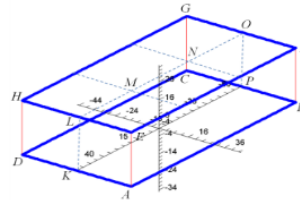

(a)

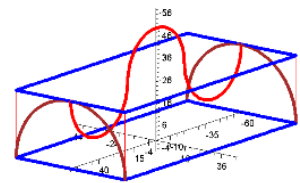

(b)

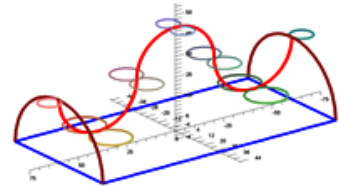

(c)

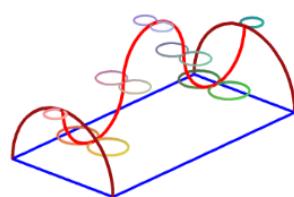

(d)

Figure 4 Rack design (flower vase).

Table 3. Plotting rack design using curves (Figure 4c,4d)

\section{Curves Formulas Using Maple of Figure}

$>$ with(plots):

$p 1:=$ spacecurve $\left(\left\{\left[(1-u)^{*} 60+u^{*}(-60),(1-u)^{*}(-30)+u^{*}(-30),(1-u)^{*} 0+u^{*} 0\right],\left[(1-u)^{*} 60+u^{*}(-60),(1-u)^{*} 30+30 * u\right.\right.\right.$, $\left.\left.(1-u)^{*} 0+u^{*} 0\right]\right\}, u=0 . .1$, thickness $=4$, color $=$ blue $)$ :

$p 2:=$ spacecurve $\left(\left\{\left[(1-u) *(-60)+u^{*}(-60),(1-u) * 30+u^{*}(-30),(1-u)^{*} 0+u^{*} 0\right],\left[(1-u)^{*} 60+60 * u,(1-u) * 30+u^{*}(-30)\right.\right.\right.$, $\left.\left.(1-u) * 0+u^{*} 0\right]\right\}, u=0 . .1$, thickness $=4$, color $=$ blue $)$ :

$t 1:=\operatorname{spacecurve}\left(\left\{\left[(20) * \cos \left(u^{*} 2 * P i\right)+40,0,20 * \sin \left(u^{*} 2 * P i\right)+30\right],\left[(20) * \cos \left(u^{*} 2 * P i\right)-40,0,20 * \sin \left(u^{*} 2 * P i\right)+30\right]\right.\right.$, $\left.\left[(20) * \cos \left(u^{*} 2 * P i-P i\right)+0,0,20 * \sin \left(u^{*} 2 * P i-P i\right)+30\right]\right\}, u=0.5 . .1$, thickness $=5$, color $=$ red $):$

$k 12:=\operatorname{spacecurve}\left(\left\{\left[-60,(30)^{*} \cos \left(u^{*} P i\right), 30 * \sin \left(u^{*} P i\right)\right],\left[60,(30)^{*} \cos \left(u^{*} P i\right), 30 * \sin \left(u^{*} P i\right)\right]\right\}, u=0 . .1\right.$, thickness $=5$, color= brown):

$C:=\operatorname{spacecurve}\left(\left\{\left[5^{*} \cos \left(u^{*} 2 * P i\right)+65,5 * \sin \left(u^{*} 2 * P i\right), 30\right],\left[5 * \cos \left(u^{*} 2 * P i\right)-65,5 * \sin \left(u^{*} 2 * P i\right), 30\right]\right\}, u=0 . .1\right.$, thickness $=4)$ :

$C 2:=$ spacecurve $\left(\left\{\left[9 * \cos \left(u^{*} 2 * P i\right)+40,9 * \sin \left(u^{*} 2 * P i\right)+9,10\right],\left[9 * \cos \left(u^{*} 2 * P i\right)+40,9 * \sin \left(u^{*} 2 * P i\right)-9,10\right]\right\}, u=0 . .1\right.$, thickness $=4)$ :

$C 3:=$ spacecurve $\left(\left\{\left[7 * \cos \left(u^{*} 2 * P i\right)+20,7 * \sin \left(u^{*} 2 * P i\right)+7,30\right],\left[7 * \cos \left(u^{*} 2 * P i\right)+20,7 * \sin \left(u^{*} 2 * P i\right)-7,30\right]\right\}, u=0 . .1\right.$, thickness $=3)$ :

$C 4:=\operatorname{spacecurve}\left(\left\{\left[5^{*} \cos \left(u^{*} 2 * P i\right)+0,5 * \sin \left(u^{*} 2 * P i\right)+5,50\right],\left[5 * \cos \left(u^{*} 2 * P i\right)+0,5 * \sin \left(u^{*} 2 * P i\right)-5,50\right]\right\}, u=0 . .1\right.$, thickness $=3)$ :

$C 5:=\operatorname{spacecurve}\left(\left\{\left[7 * \cos \left(u^{*} 2 * P i\right)-20,7 * \sin \left(u^{*} 2 * P i\right)+7,30\right],\left[7 * \cos \left(u^{*} 2 * P i\right)-20,7 * \sin \left(u^{*} 2 * P i\right)-7,30\right]\right\}, u=0 . .1\right.$, thickness $=3)$ :

$C 6:=$ spacecurve $\left(\left\{\left[9 * \cos \left(u^{*} 2 * P i\right)-40,9 * \sin \left(u^{*} 2 * P i\right)+9,10\right],\left[9 * \cos \left(u^{*} 2 * P i\right)-40,9 * \sin \left(u^{*} 2 * P i\right)-9,10\right]\right\}, u=0 . .1\right.$, thickness $=4)$ :

$\operatorname{display}(p 1, p 2, t 1, k 12, C, C 2, C 3, C 4, C 5, C 6$, view $=[-75 . .75,-45 . .45,-5$.. 65]);

Modeling handicraft and home industries objects with surfaces need to recognize various component features, the measure, the position of the parts, and the aesthetic of the goods [19-21]. In this case, Equation 7-13 and some modifications formula provide some facilitates to create the shapes and the model of the goods using stages as follows. First, we determine each position of the part object and its measures from bottom to top, from back to front, or from left to right, consecutively. Second, connecting some surfaces of Equation 7-13 using a Maple suitable with the arranged positions will create the shape of the part of the goods. Finally, showing naturally, the designed objects can use the Maple menu related to the presentation style of surfaces, color, or lighting. For example, implementing the formula cone and paraboloid (Equation 11,10) ordered from bottom to top can model a hat shape (Figure 5a). In the same manner, it can create a model of Figure $5 \mathrm{~b}$ and Figure 5c. Moreover, the application of many Equations 7-13 gives the complex shapes as illustrated in Figures $5 \mathrm{~d}, 5 \mathrm{e}$, and 5f. Table 4 shows some equations used to draw Figures $5 \mathrm{a}, 5 \mathrm{~b}$, and 5d using software-Maple.

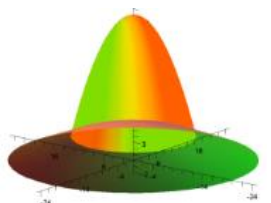

(a)

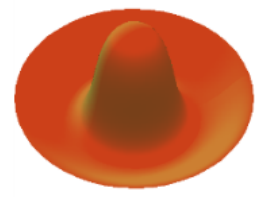

(c)

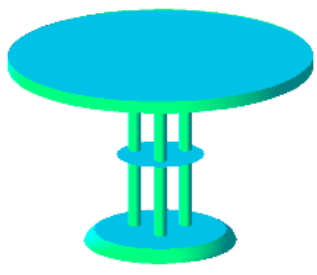

(e)

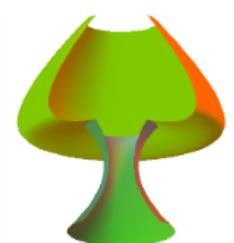

(b)

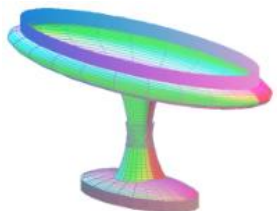

(d)

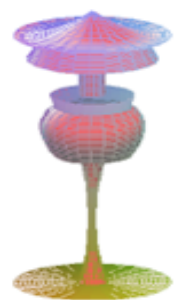

(f)
Figure 5 Simulation results of the object design. 
Table 4. Objects design constructed with surfaces

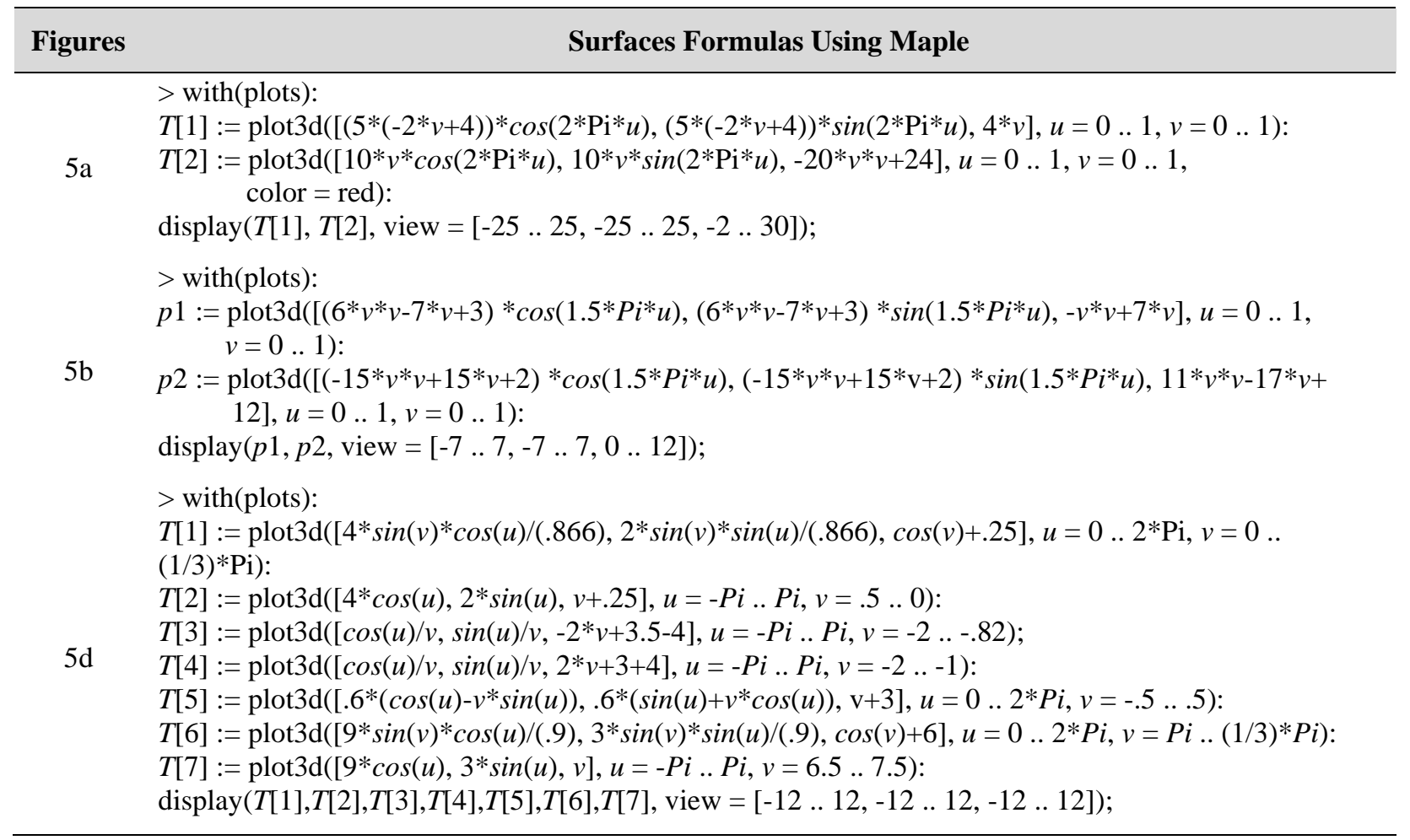

\subsubsection{Pipes Design}

Consider two perpendicular unity constant vectors $\mathbf{v}_{1}$ and $\mathbf{v}_{2}$ with $\mathbf{v}_{1} \wedge \mathbf{v}_{2}=\mathbf{v}$ and the parametric curve $\gamma(v)$ in the form of Equation (4). On the other side, consider a curve $\mathbf{C}(u)$ with their normal and binormal vectors $\mathbf{n}$ and b shown in Figure 6a. It can define a tube with a center of the curve, i.e., a line or the curve $\mathbf{C}(u)$ and the crosssection curve of the pipe $Y(v)$ in this way [3,5]

$$
\begin{aligned}
& \mathbf{T}_{v}(u v)=\left[\begin{array}{c}
\left.\left\langle\mathrm{x}_{\mathrm{C}}, \mathrm{y}_{\mathrm{C}}, z_{C}\right\rangle+\lambda u . \mathbf{v}\right]+ \\
\Upsilon(v) \cdot\left[\cos (\varphi) \mathbf{v}_{1}+\sin (\varphi) \mathbf{v}_{2}\right]
\end{array}\right. \\
& \mathbf{T}(u, v)=\mathbf{C}(u)+\Upsilon(v) \cdot[\cos (\varphi) \mathbf{b}+\sin (\varphi) \mathbf{n}]
\end{aligned}
$$

with $\lambda$ parameter constant, the value $\varphi=2 \pi v$ and $0 \leq u, v$ $\leq 1$. For example, if the unity constant vectors $\mathbf{v}_{1}=$ $\langle 0,0,1\rangle, \mathbf{v}_{2}=\langle 0,1,0\rangle,\left\langle\mathrm{x}_{\mathrm{C}}, \mathrm{y}_{\mathrm{C}}, z_{C}\right\rangle=\langle 5,0,0\rangle$ and $\gamma(v)=$ $2+\cos (6 \pi v)$, then we find Figure $6 \mathrm{~b}$. When the center curve of pipe $\mathbf{C}(u)=<17 u^{3}-30 u^{2}+6 u-12,-39 u^{3}+51 u^{2}$ $-36 u+10,203 u^{3}+393 u^{2}-195 u+15>$ and $Y(v)=3$, using Equation (6) and Equation (15) will obtain the tube shape in Figure 6c, 6d.

\section{CONCLUSIONS}

This study develops learning materials to apply the parametric formulas of curves and surfaces to design the shape of cottage industry, pipes shape and modeling the handicrafts with software-Maple. In modeling these goods, software-Maple helps the students recognize and implement the curves and surfaces formula, visualize and present the industrial objects naturally. Moreover, aiding Maple, the collegers learn creatively, visually, and responsibly with their knowledge, technological skills, and experiences in implementing the differential geometry formulas (especially the curves and surfaces) for modeling industrial objects.

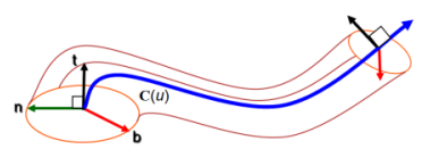

(a)

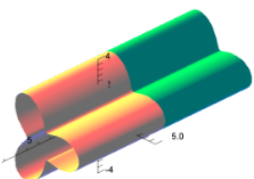

(b)

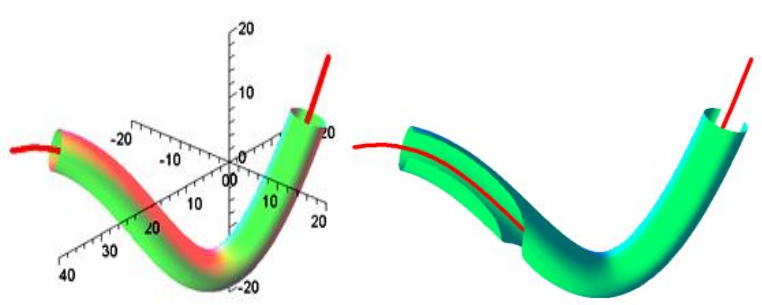

(c)

(d)

Figure 6 Pipes construction. 


\section{REFERENCES}

[1] M.E. Mortenson, Geometry Modeling, Jonh Wiley and Sons Inc., New York, 1996.

[2] J. Oprea, Differential Geometry and its Applications, The Mathematical Association of America, Washington DC, 2007.

[3] Kusno, On the modeling of cross-section and longitudinal section of pipes, Journal of Physics Conference Series, vol. 321(2), 2019, pp. 1-8. DOI: https://doi.org/10.1088/1742-6596/1321/2/022068

[4] Kusno, Modeling pipes using pipes' center curves of quadratic and cubic spline interpolation, AIP Conference Proceedings, vol. 2329, 2021. DOI: https://doi.org/10.1063/5.0042248

[5] Kusno, A. C. Prihandoko, Continuous connection of two adjacent pipe parts defined by line, Bézier and Hermit center curves, Journal of Physics Conference Series, vol. 1008(1), 2018, pp. 1-7. DOI: https://doi.org/10.1088/1742-6596/1008/1/ 012005

[6] Kusno, Modeling of developable surfaces using Hermite spline interpolation curves, Advances in Mathematics: Scientific Journal, vol. 9(10), 2020, pp. 8431-8442. DOI: https://doi.org/10.37418/ amsj.9.10.72

[7] Kusno, Fitting a curve, cutting surface, and adjusting the shapes of developable Hermite patches, Mathematics and Statistics, vol. 8 (6), 2020, pp. 740-746. DOI: https://doi.org/10.13189/ ms.2020.080615

[8] Kusno, On the modeling of developable hermite patches, Journal of Mathematical and Computational Science, vol. 11(2), 2021, pp.11451165. DOI: https://doi.org/10.28919/jmcs/5293

[9] F. Yamaguchi, Curves and Surfaces in Computer Aided Geometric Design, Springer-Verlag, Berlin, 1988.

[10] S. Montiel, A. Ros, Curves and Surfaces, American Mathematical Society, Providence, 2009.

[11] M. L. Abell, J. P. Braselton, Maple by Example, Elsevier Inc, Amsterdam, 2005.

[12] A-K. Carstensen, J. Bernhard, Design Science Research - an engineering research approach to improve methods for engineering education research, Research in Engineering Education Symposium (REES), 2015, pp. 1-9. https:// www.researchgate.net/publication/280611224
[13] A-K. Carstensen, J. Bernhard, Design science research - a powerful tool for improving methods in engineering education research, European Journal of Engineering Education, vol. 1-18, 2018. https://doi.org/10.1080/03043797.2018.1498459

[14] R. C. Richey, J. D. Klein, Developmental Research Methods: Creating Knowledge from Instructional Design and Development Practice, Journal of Computing in Higher Education Spring, vol. 16(2), 2005, pp. 23-38.

[15] R. C. Richey, J. D. Klein, Design and Development Research, Handbook of Research on Educational Communications and Technology, vol. (141-150), Springer Science+Business Media New York, 2014. DOI: 10.1007/978-1-4614-3185-5_12

[16] Institute of Education Sciences (IES), Common Guidelines for Education Research and Development, A Report from the Institute of Education Sciences, U.S. Department of Education and the National Science Foundation, 2013, Retrieve 17th July 2021 from https://ies.ed.gov/pdf/ commonguidelines.pdf

[17] M. B. Monagan, K. O. Geddes, K. M. Heal, G. Labahn, S. M. Vorkoetter, J. McCarron, P. DeMarco, Maple 7 Programming Guide, Waterloo Maple Inc, Canada, 2001.

[18] Maplesoft, User Manual, Waterloo Maple Inc, Canada, 2021.

[19] Kusno, I. H. Agustin, I. Halikin, On the modeling of the object surface reliefs of marble handicrafts using quartic curves and circles, IOP Conf. Series: Earth and Environmental Science, vol.243, 2019. DOI: https://doi.org/10.1088/1755-1315/243/1/ 012017

[20] W.T. Li, M.C. Ho, C. Yang, A Design ThinkingBased Study of the Prospect of the Sustainable Development of Traditional Handicrafts, Sustainability, vol. 11(18), 2019, pp. 1-26. DOI: https://doi.org/10.3390/su11184823

[21] J. Fengfan, H. Yue, Traditional Tie-dye Handicraft and Modern Design Concept Combined, Journal of Arts \& Humanities, vol. 06(8), 2017, pp. 12-15. DOI: http://dx.doi.org/10.18533/journal.v6i9.1230 